NIST Special Publication 1267

\title{
Longitudinal Study of Complex Event Resilience of Small- and Medium-Sized Enterprises: Natural Disaster Planning and Recovery During the COVID-19 Pandemic (Wave 2)
}

\author{
Jennifer F. Helgeson ${ }^{1}$, Juan F. Fung ${ }^{1}$, Alfredo R. Roa Henriquez ${ }^{1}$, \\ Ariela Zycherman ${ }^{2}$, Claudia Nierenberg ${ }^{2}$, David T. Butry ${ }^{1}$, Donna Ramkissoon ${ }^{1}$, Yating Zhang ${ }^{1}$ \\ ${ }^{1}$ Applied Economics Office, National Institute of Standards and Technology \\ ${ }^{2}$ Climate Program Office, National Oceanic and Atmospheric Administration
}

May 2021

\section{Executive Summary}

Researchers at NIST and NOAA launched a longitudinal effort to assess Complex Event Resilience of Smalland Medium-sized Enterprises (SMEs) in May 2020. Initial data collection took place during summer 2020. This publication reports upon initial findings from the second wave of data collection, which took place between December 2020 and February 2021. This effort addresses the gap in research on the experiences of SMEs dealing with complex events generally and those that arise during a pandemic, specifically.

Initial findings from the panel of respondents who responded to the Wave 2 survey, which covers selfreported experiences during the period August 1, 2020 to February 1, 2021, include:

- $31.6 \%$ of respondents experienced at least one natural disaster during this period.

- $68.8 \%$ of respondents implemented some adaptation/coping strategies for COVID-19, with $26 \%$ reporting the use of natural hazard mitigation strategies.

- $\quad 28.7 \%$ of respondents plan to adopt practices used during the COVID-19 pandemic in anticipation of future natural hazards.

- $\quad 75.3 \%$ of businesses are concerned about future complex events, of which $45.2 \%$ identify their concern as arising from natural disaster(s) and a subsequent wave of COVID-19 associated restrictions.

There is evidence that respondents have learned and adapted during the course of the pandemic between Wave 1 and Wave 2 data collections. The four main categories of resources and information identified in Wave 2 by respondents as areas that could help them address complex event risks that may arise during the COVID-19 transmission period and into the recovery period, while facing potential additional complex events include: (1) Assistance navigating deep uncertainty, (2) Clear, detailed information and training, (3) Assistance with personal protective equipment (PPE) and other equipment, and (4) Access to financial assistance and financial information.

\section{Key words}

Adaptation, climate, community resilience, complex event, coping, COVID-19, extreme weather events, natural disaster, hazard, longitudinal, mitigation, online survey, pandemic, resilience planning, recovery, risk mitigation, small- and medium-sized enterprise, small business, survey instrument, vulnerable.

Certain commercial entities, equipment, or materials may be identified in this document in order to describe an experimental procedure or concept adequately. Such identification is not intended to imply recommendation or endorsement by the National Institute of Standards and Technology, nor is it intended to imply that the entities, materials, or equipment are necessarily the best available for the purpose. This publication is available free of charge: https://doi.org/10.6028/NIST.SP.1267 


\section{Background and Motivation}

Small- and medium sized enterprises (SMEs) make up $99.9 \%$ of U.S. businesses and employed 59 million people in 2018 (SBA, 2019). COVID-19 (Coronavirus Disease 2019) has greatly impacted SME functionality and required adaptation and coping as circumstances continued to change relative to safety measures that limited customer interactions and reduce employee availability, as well as through supply chain disruptions. As of February 1, 2021, total small business revenue had declined $30.9 \%$ relative to January 2020, driven in large part by reduced consumer spending. ${ }^{1}$ As Chetty et al. $(2020)^{2}$ observe, the revenue losses resulted in unemployment, with low-wage workers more likely to lose their jobs. SMEs in areas vulnerable to extreme weather and climate face potential complex events as they prepare for or react to natural disasters during the pandemic. Furthermore, moving towards recovery from these impacts can be particularly daunting, especially for SME operators that are most socially vulnerable and find themselves needing to plan for the potential for additional natural hazards.

Researchers at NIST and NOAA launched a longitudinal effort to assess Complex Event Resilience of SMEs in May 2020. Initial data collection took place during summer 2020 and is reported in Helgeson et al. $(2020)^{3}$. This report presents initial findings from the second wave of data collection between December 2020 and February 2021 with respondents answering for the time period beginning August 1, 2020. ${ }^{4}$ The online survey was open between January 28 and February 26, 2021 and addressed the following themes:

1. Current SME experience with COVID-19;

2. Current SME experience with natural disasters during the COVID-19 pandemic;

3. Future plans to cope with COVID-19 and natural disasters; and

4. Future planning for natural disasters and other complex event types.

\section{Methods}

Survey instrument. The survey ${ }^{5}$ asked respondents about how COVID-19 has continued to impact their SME, and how the SME is adapting to and/or coping with these impacts; about the SME's experience with natural disasters and resilience planning in the context of COVID-19; and the respondent's expectations about the future of the SME, generally and relative to a number of potential complex event types. In addition, the survey collected demographic information and SME background information. Respondents were asked to indicate if they would like to be contacted again in the future and if they would like to be sent a report on the Wave 2 survey findings. For more details on the survey development and to view the instrument, see NIST DCI 003 (Helgeson et al., 2021a). ${ }^{6}$

\footnotetext{
1 In fact, revenue in low-income zip codes declined $26.1 \%$ and revenue in high-income zip codes declined 37.2\%, relative to January 2020. Chetty et al. (2020). The Opportunity Insights Economic Tracker. https://tracktherecovery.org/. Accessed: 2021-03-29.

${ }^{2}$ Chetty, R., Friedman, J. N., Hendren, N., Stepner, M., \& The Opportunity Insights Team. (2020). The economic impacts of COVID-19: Evidence from a new public database built using private sector data (No. w27431). National Bureau of Economic Research.

${ }^{3}$ Helgeson, J.F., J.F. Fung, Y. Zhang, A.R. Roa Henriquez, A. Zycherman, C. Nierenberg, D.T. Butry, D. Ramkissoon. (2020). Complex Event Resilience of Small- and Medium-Sized Enterprises: Natural Disaster Planning During the COVID-19 Pandemic, NIST SP 1258. https://doi.org/10.6028/NIST.SP.1258.

${ }^{4}$ Responses were collected between Dec 8, 2020 and February 22, 2021. Respondents provided answers for the period after August 1.

${ }^{5}$ The survey "Wave 2 SME Recovery from a Pandemic in the Face of Natural Hazard Risks" is approved by the NIST IRB and under OMB CONTROL NO. 0693-0078 Expiration Date 07/31/2022.

${ }^{6}$ Helgeson, J.F., J.F. Fung, Y. Zhang, A.R. Roa Henriquez, A. Zycherman, C. Nierenberg, D.T. Butry, D. Ramkissoon. (2021a). Eliciting lessons from small- and medium-sized enterprises for resilience during and following complex events: longitudinal data collection (Wave 2).

https://doi.org/10.6028/NIST.DCI.003
} 
Sample. Invitations to complete the Wave 2 online survey reached SMEs through direct emails. All respondents had opted into being contacted for potential participation in Wave 2 at the end of the Wave 1 survey. Additional details about the initial sample can be found in Helgeson et al. (2020a).

\section{Descriptive Statistics}

From the 1374 total Wave 1 survey responses, 705 respondents indicated that they would like to be contacted again. A total of 247 responded to the Wave 2 follow-up survey, for a $35 \%$ response rate. The distribution of respondents across Census regions was similar to the overall Wave 1 sample; see Figure 1. Moreover, about $50 \%$ of respondents self-identify as operating an essential SME, which corresponds to the value
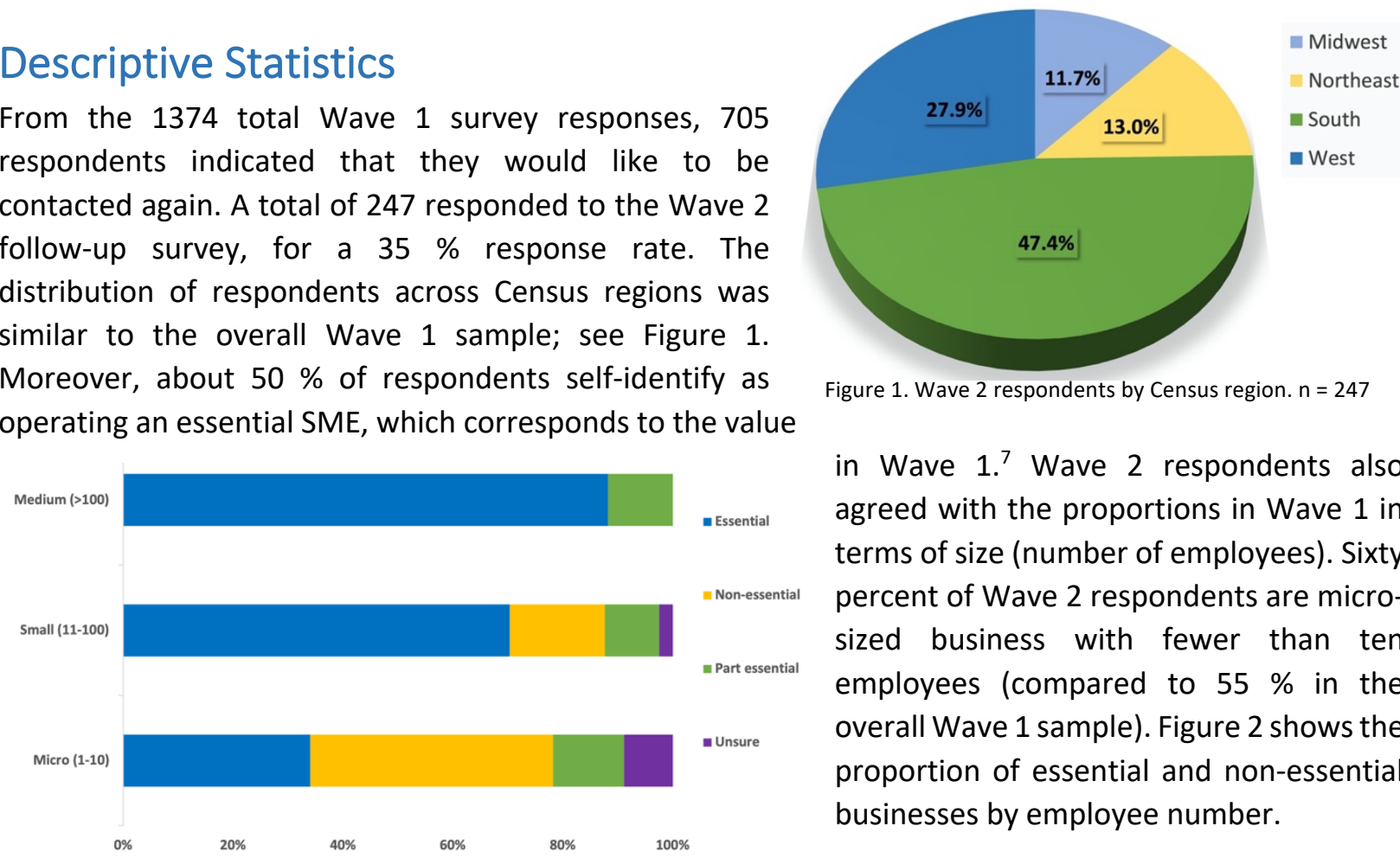

Figure 1. Wave 2 respondents by Census region. $n=247$

in Wave $1 .^{7}$ Wave 2 respondents also agreed with the proportions in Wave 1 in terms of size (number of employees). Sixty percent of Wave 2 respondents are microsized business with fewer than ten employees (compared to $55 \%$ in the overall Wave 1 sample). Figure 2 shows the proportion of essential and non-essential businesses by employee number.

Figure 2. Wave 2 respondents by size and self-reported essential designation. $n=245$

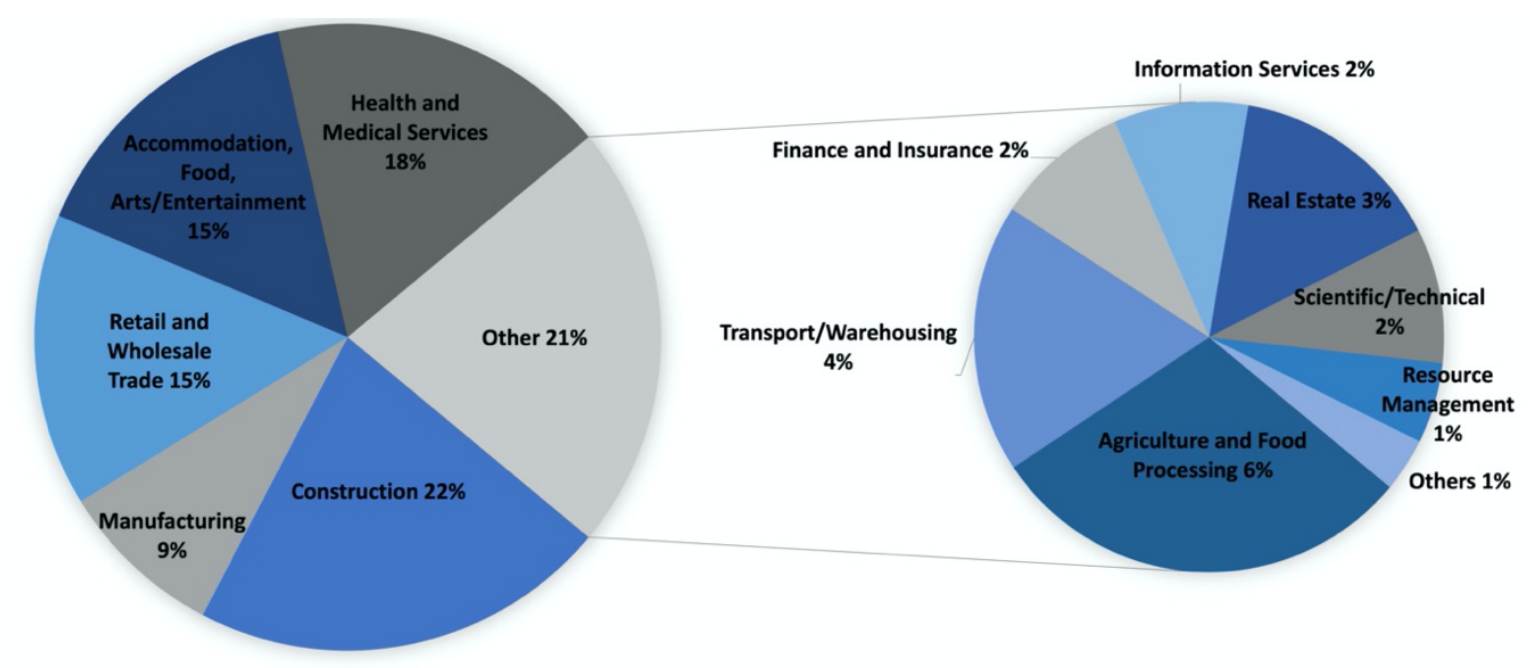

Figure 3. Wave 2 respondents by sector. $n=246$

\footnotetext{
${ }^{7}$ The survey did not define "essential" for respondents; thus, respondents self-identified whether their SME was deemed essential in their state. Guidance issued by the Department of Homeland Security's Cybersecurity and Infrastructure Security Agency ("CISA Guidance") identified 16 industry sectors considered "essential critical workforce infrastructure." See: https://www.cisa.gov/sites/default/files/publications/CISAGuidance-on-Essential-Criticallnfrastructure-Workers-1-20-508c.pdf
} 
The breakdown of respondents in Wave 2 by sector is provided in Figure 3; the sectoral division aligns with the division in the overall Wave 1 sample. Wave 2 respondents' demographic breakdown is as follows: $33 \%$ self-report as representing a woman-owned business, while $16 \%$ identify as minorityowned, and $6 \%$ identify as Veteran-owned. The panel data is slightly skewed with relatively more womenowned business operators answering in Wave 2 than in the overall Wave 1 sample.

Finally, Wave 2 respondents represented single owner businesses (36\%), businesses held as a partnership (11\%), corporation subsidiaries (50\%), and other ownership types (3\%).

\section{Observations}

Key initial findings from the analysis of quantitative and qualitative survey data are presented in this section.

1. Past SME natural disaster experience. In Wave 1, a majority of panel respondents in each region indicated that their SME has experienced a natural disaster ${ }^{8}$ in the past ten years, as well as during the period March 13-August 1, 2020. The percent of SME operators reporting this experience within each Census region: 77.8 \% (Midwest), $92.3 \%$ (Northeast), $91.9 \%$ (South), and $78.2 \%$ (West).

\section{Recent SME experience with COVID-19.}

The survey asked respondents about the current status of their business. Of those who responded, 11.3 $\%$ indicated that their SME is either temporarily (10.5\%) or permanently closed $(0.8 \%)$. Figure 4 shows the self-reported impact of the pandemic on the SME (not including those permanently closed): $5 \%$ report no impact, $3 \%$ report that the impact is just starting, $9 \%$ report that the impact is declining, $1 \%$ report that the impact is over, $7 \%$ report a positive impact, and $74 \%$ report continued (negative) impact.

The survey asked respondents to indicate the most important factors that influenced their choice of whether to halt, resume, or continue SME operation. The answer options for this question are not mutually exclusive. These decisions were largely informed by general employee health and well-being (43\%) and concern of infection for customers and those to whom services are delivered (48\%). The most frequently selected factors all relate directly or indirectly to health concerns, such as

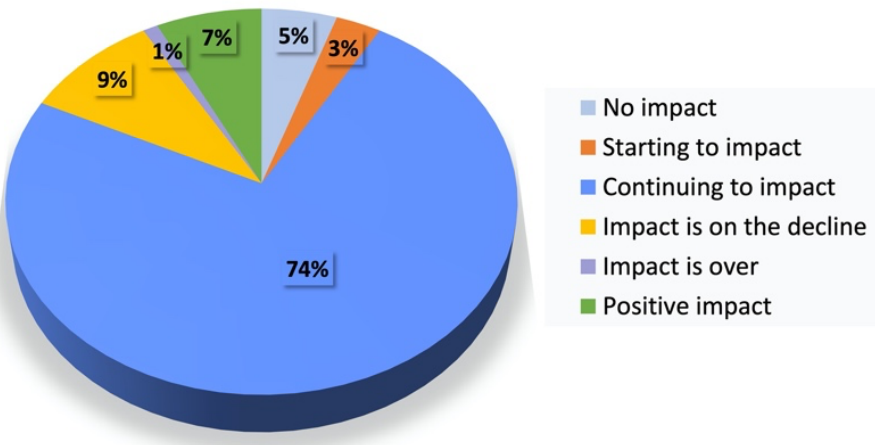

Figure 4. Reported current impact of COVID-19 on SMEs. $n=191$ availability of PPE and/or cleaning supplies (28\%), local business opening guidance/regulations (23\%), and local government suggestions (non-regulatory) (22\%). Furthermore, issues related to SME inputs, labor, and outputs of the business were also highly rated: disruption to supply and inventory delivery (21 $\%)$, business margins ( $23 \%)$, and the staff's desire to return to work ( $21 \%)$.

\footnotetext{
${ }^{8}$ Either acute or chronic. A chronic risk is one that is recurring, can often be expected, and for which an SME may plan for regularly. Chronic natural disaster risks include drought, extreme cold, heat waves, winter storms, flooding. Acute risks are associated with less predictability and are often defined by low-probability and high-impact. Acute natural disaster risks include hurricanes, storm surge, earthquakes, tsunamis, tornadoes, and wildfires.
} 
There is evidence of adaptive learning across panel responses in Wave 2; respondents report learning to adapt to the evolving uncertainty surrounding COVID-19 associated (mandated and voluntary) limitations on SMEs. In particular, in Wave 1, those SME operators who self-identified their business as essential do not appear to have been better prepared than their non-essential counterparts for a pandemic. This seems to be the case for Wave 2 respondents as well. However, some non-essential business operators seem to have been able to use the time during mandatory closures and mandated limits on their activities to learn from their and surrounding businesses' experiences to plan more effectively than businesses with no reported down time during the pandemic. However, there are limitations posed by both the type of

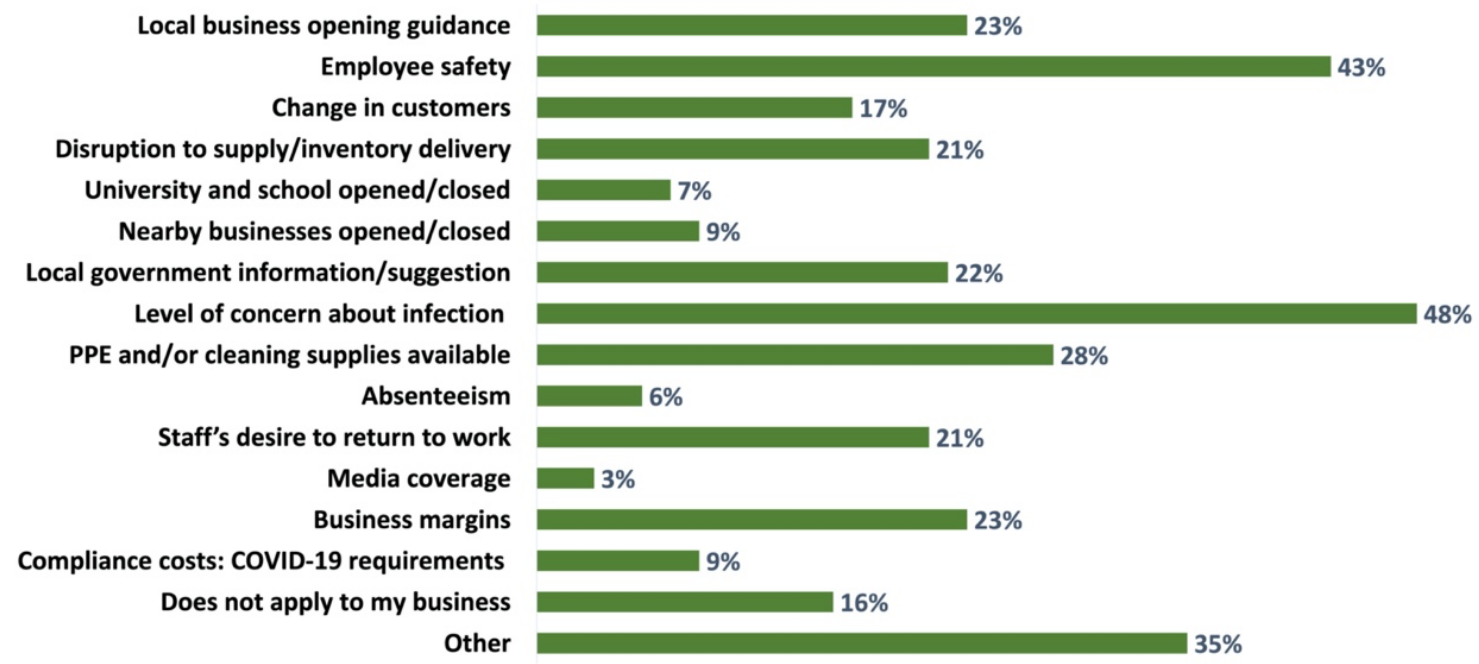

Figure 5. Reported factors influencing the choice of whether or not to resume operations. $n=193$

goods and services typically provided by an SME and the current resources available, which limited the flexibility of the SME's response. Indeed, $50 \%$ of respondents reported at least $10 \%$ of their monthly expenses went to payments for expenses that no longer generated revenue. This includes structural changes required by regulations (e.g., dividers in office and restaurant facilities) and nonstructural additions like increased programs to provide support to employees and the community, for which the SME received no additional revenue. Those respondents who still had the option to make additional cuts reported continuing to reduce staffing to the lowest level reasonable, reduced pricing to consumers (for in-person services in particular), reduced wages - for themselves ahead of their employees - and "worked with peer groups to come up with ideas to survive the economic disaster."

Supply chain issues seem to have lessened due to lack of inventory; however, access to supplies is still a problem for some, as up-chain suppliers limit contracts to larger mid-process manufacturers and cause cascading impacts to more micro-sized operations. In particular one small manufacturer noted that largescale suppliers reduced credit limits and prioritized larger companies when allocating limited shipments throughout the pandemic to protect their business margins.

Financial difficulties arising from a number of direct and indirect COVID-19 specific impacts were frequently discussed. Many micro-sized enterprises that reported continuing to be (negatively) impacted express concerns over continuing to pay employees' salaries and feel as though they are "out of options." There was continued acknowledgement of the assistance requested and received; however, it was 
common to see responses noting that they "received the PPP loan in April along with $100 \%$ forgiveness," but "still had to dip into savings to supply cash flow."

Respondents were asked if actions taken to prepare their SME for natural disasters in the past helped in preparation for or to cope with the impacts of COVID-19. Out of 165 responses, $38.2 \%$ indicated that disaster preparedness has helped them with COVID-19, while $52.1 \%$ said it has not, and $9.7 \%$ report being "unsure." These values are relatively higher than those reported for this question in the Wave 1 survey. This may arise from (1) self-selection biases of those who answered the Wave 2 survey, (2) relatively greater complex events from natural disasters occurring during the time period covered by

Table 1. Respondents indicating whether natural disaster preparedness helped their SME prepare for or cope with COVID-19 impacts. $n=138$

\begin{tabular}{|c|c|c|}
\hline $\begin{array}{l}\text { Actions to address natural } \\
\text { disasters in the past helped } \\
\text { address COVID-19 impacts }\end{array}$ & $\begin{array}{c}|c| \\
\text { Natural disaster experience } \\
\text { reported }\end{array}$ \\
\hline & NO & YES \\
\hline YES & $11 \%$ & $89 \%$ \\
\hline NO & $14 \%$ & $86 \%$ \\
\hline Unsure & $23 \%$ & $77 \%$ \\
\hline
\end{tabular}

Wave 2, and/or (3) the need for SME operators to apply more strategies as the COVID-19 transmission period goes on. Given the large percentage of respondents who experienced a natural hazard that impacted their SME during the Wave 2 response period, it is hard to disentangle the extra burden placed upon respondents by complex events from their answer about experiences adapting to and coping with COVID-19 impacts. Table 1 presents results conditional on experience with natural disasters in the past ten years, as reported in Wave 1.

\section{Recent SME experience with natural disasters during the COVID-19 Pandemic. The survey} asked respondents if their SME had experienced a natural disaster event since August $1,2020 .{ }^{9}$ Of those who responded, $40.5 \%$ had experienced at least one such event; of these, $33.2 \%$ had experienced such an event with adverse impacts and $7.3 \%$ had experienced such an event with no reported adverse impacts on the SME (Figure 6). These numbers are not surprising given the extent of extreme weather events and natural disasters reported in the latter half of 2020 (NOAA, 2021)..$^{10}$ Of those impacted by a natural disaster during this period, $33.8 \%$ indicated that their institutional response to the event was affected by COVID-19; see Figure 7.

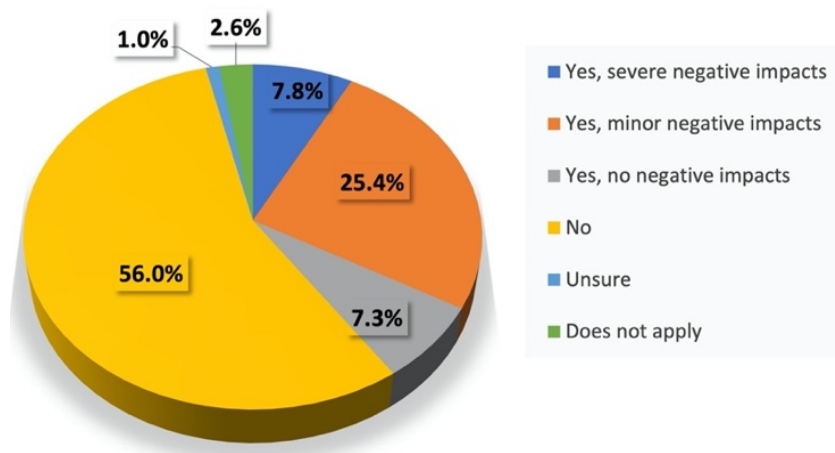

Figure 6. SMEs affected by a natural disaster during COVID-19 between August 1, 2020 and February 26, 2021. $n=193$

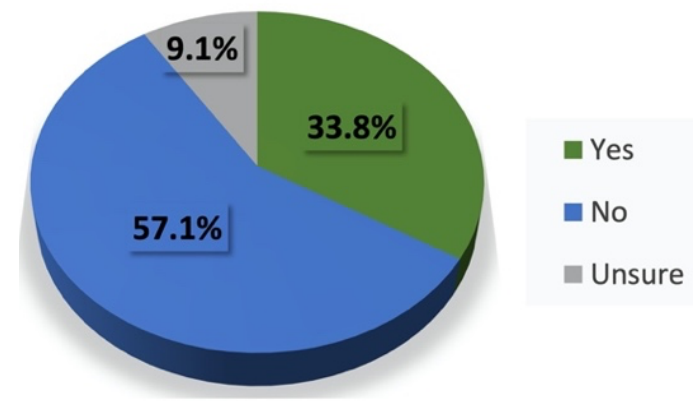

Figure 7. Figure 7. Impact of COVID-19 impacts on reported SME response to natural disaster. $n=94$

\footnotetext{
${ }^{9}$ Wave 1 asked respondents if they had experienced a natural disaster since March 13, 2020, which was the date of the Federal Emergency Declaration. See: "Proclamation on Declaring a National Emergency Concerning the Novel Coronavirus Disease (COVID-19) Outbreak." https://www.whitehouse.gov/presidential-actions/proclamation-declaring-national-emergency-concerning-novel-coronavirus-disease-covid19-outbreak/. Wave 1 data collection closed at the end of July 2020; hence, Wave 2 asks about experience from August 1, 2020.

10 National Oceanic and Atmospheric Administration. "Record number of billion-dollar disasters struck U.S. in 2020."January 8, 2021. https://www.noaa.gov/stories/record-number-of-billion-dollar-disasters-struck-us-in-2020. Accessed March 30, 2021.
} 
Some $27 \%$ of respondents indicated that COVID-19 has left them unable to cope with a natural hazard should one occur in the next twelve months.

Even respondents who indicated no direct impact from COVID-19 on their natural disaster response indicated that they were indirectly affected by decisions they had previously made to address COVID-19 impacts on the SME, such as the use of financial reserves otherwise earmarked for natural disaster preparedness. Across sectors and designation of SMEs as essential

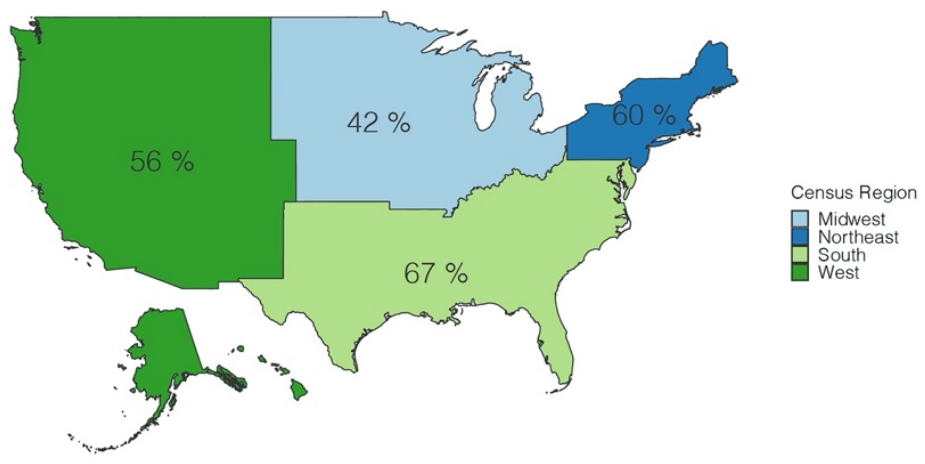

Figure 8. Fraction of respondents in each Census region that expressed concern over natural disaster risk in the context of recovery from COVID-19. $n=186$ natural disaster hit in addition to the pandemic.

\section{Future plans: towards recovery and future complex event impacts}

Assessing Complex Event Responses. The survey asked respondents to consider their response to COVID-19 and the complex event conditions experienced so far. In particular, respondents were asked whether they would have made different decisions for their business if they had the information they have today. Respondents readily acknowledge the extent of the uncertainty and novelty surrounding COVID-19 conditions, as it started like an acute event and has continued as a chronic condition. Of the 196 who responded to this question, $29.6 \%$ indicated that they would have definitely made other choices, $29.1 \%$

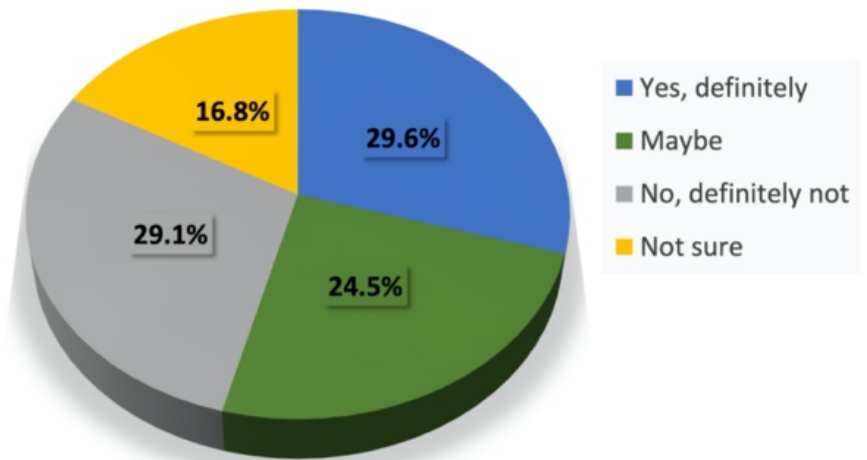

Figure 9. Fraction of respondents who indicate whether they would have made different decisions at the start of the COVID-19 pandemic if they had the information available in early February 2021. $\mathrm{N}=196$. indicate that they would not have, and $41.3 \%$ indicate that they are unsure/may have made other choices; see Figure 9. For those who would have made other choices, many of the respondents indicate that they would have made more extreme reductions in services, staffing, and inventory at the start of the pandemic - frequently with the aim of maintaining greater flexibility with liquidity as COVID-19 conditions persisted. Those who indicated a no regrets approach appear to feel that they were able to adjust with agility since the start of the pandemic, with one respondent remarking, "we've done it right from the beginning...[we had] policies in place with our employees that ensure that we all stay healthy while working together within suggested guidelines."

Finally, the survey asked about respondents' perception of how long it would be before the SME returns to its pre-COVID-19 business capacity. Of the 168 who responded to this question, $34.5 \%$ expected recovery in one year or less, $38.7 \%$ expected recovery in more than one year, and $3.6 \%$ expected no recovery. In addition, $13.7 \%$ expressed being already recovered. 
Future Complex Event Concerns. Looking forward, ${ }^{11}$ the survey asked respondents about their concerns regarding continued COVID-19 impacts on their SME's recovery process. In particular, respondents were asked to note their level of concern about complex events arising during the duration of the pandemic and into their recovery period. All Wave 2 respondents expressed concern over the risk of one or more complex event(s); see Table 2 for complex event types. Notably, those who had experienced or have prepared for natural disasters more readily expressed concern over a complex event across all categories. Out of 186 respondents, $50.5 \%$ reported specific concern over complex events related to the occurrence of a natural disaster during the

COVID-19 pandemic.

Figure 8 presents the fraction of respondents in each region that express such a concern.

Of those who indicated concern for a future complex event, $45 \%$ believed that they had the resources needed to protect their SME against the issues indicated; $34 \%$
Table 2. Percent of respondents in Wave 2 indicating a complex event type as a top concern, grouped by Census region and experience with a natural disaster during the pandemic.

\begin{tabular}{|l|c|c|c|}
\hline \multicolumn{1}{|c|}{ Complex Event Concern Types } & $\begin{array}{c}\text { No disaster } \\
\text { experience }\end{array}$ & $\begin{array}{c}\text { Disaster } \\
\text { experience }\end{array}$ & $\begin{array}{c}\text { Number } \\
\text { of SMEs }\end{array}$ \\
\hline Natural Hazard Concerns & $10.6 \%$ & $89.4 \%$ & 94 \\
COVID-19 Specific Concerns & $10.7 \%$ & $89.3 \%$ & 112 \\
Business Financial, Market Concerns & $11.9 \%$ & $88.1 \%$ & 118 \\
Other Health Concerns & $17.3 \%$ & $82.7 \%$ & 52 \\
Workforce Concerns & $12.4 \%$ & $87.6 \%$ & 97 \\
Consumer Concerns & $14.3 \%$ & $85.7 \%$ & 70 \\
\hline
\end{tabular}
believed that they did not, and $21 \%$ were unsure; see Figure 10.

Respondents were asked to indicate the implications of their COVID-19 experience on planning for future natural hazard impacts on their SME; $36.4 \%$ indicate that this planning will be harder, $12.7 \%$ believe it will be easier, and $41.2 \%$ believed it will remain unchanged; see Figure 11 . Furthermore, the more general an SME's disaster preparedness and response plans are (i.e., widely applicable across event types), the more the respondent perceives that such planning was applicable to the current pandemic and is flexible in addressing future disaster situations.

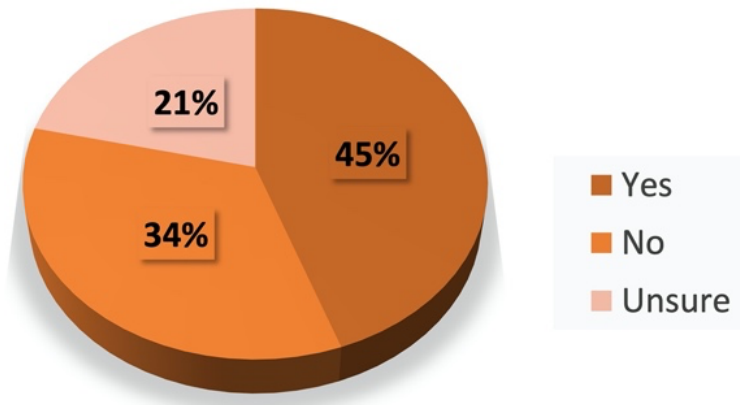

Figure 10. Of those concerned about a future complex event, fraction who believe they have the resources needed. $n=164$

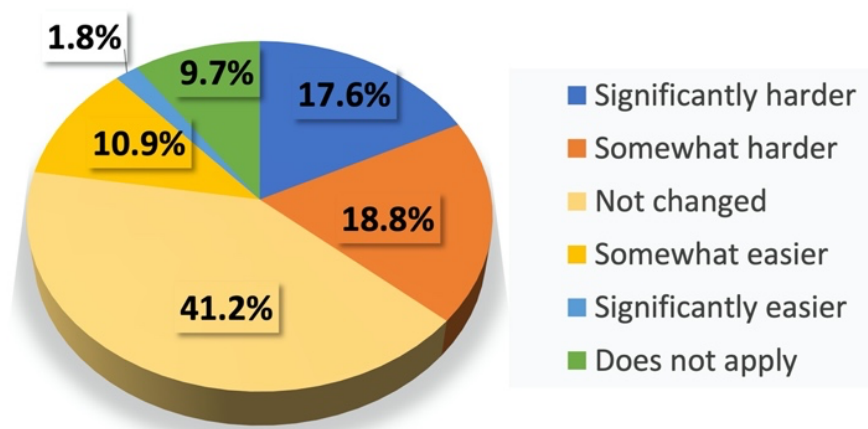

Figure 11. Reported implication of COVID-19 impacts on planning for future natural hazards. $n=165$

\footnotetext{
${ }^{11}$ This question was asked about the "future" generally, opposed to events that may have already occurred. SMEs that experienced a natural disaster during the COVID-19 pandemic up until the point of taking this survey did not necessarily indicate concern about a natural disaster in this forward-looking question.
} 
Expressed Needs. The themes of expressed needs by SME operators in this survey coincide with those identified in Wave 1. The four high-level categories identified include: (1) assistance navigating deep uncertainty, (2) clear, detailed information and training, (3) assistance with PPE and equipment, and (4) access to financial assistance and financial information. Details of how SME operators suggested addressing each of these goals are noted in Figure 12. Illustrative responses include: (1) "Who couldn't use more help/expertise? It would be great to have a marketing specialist on staff to manage the website/increase public awareness...and to build/manage online sales. We all have our plates full. We can't really do anything about limited supplies and inventory;" and (2) "Why is applying for help so complicated? There is enough stress and uncertainty rite now with complicated system upload requirements and rejection."

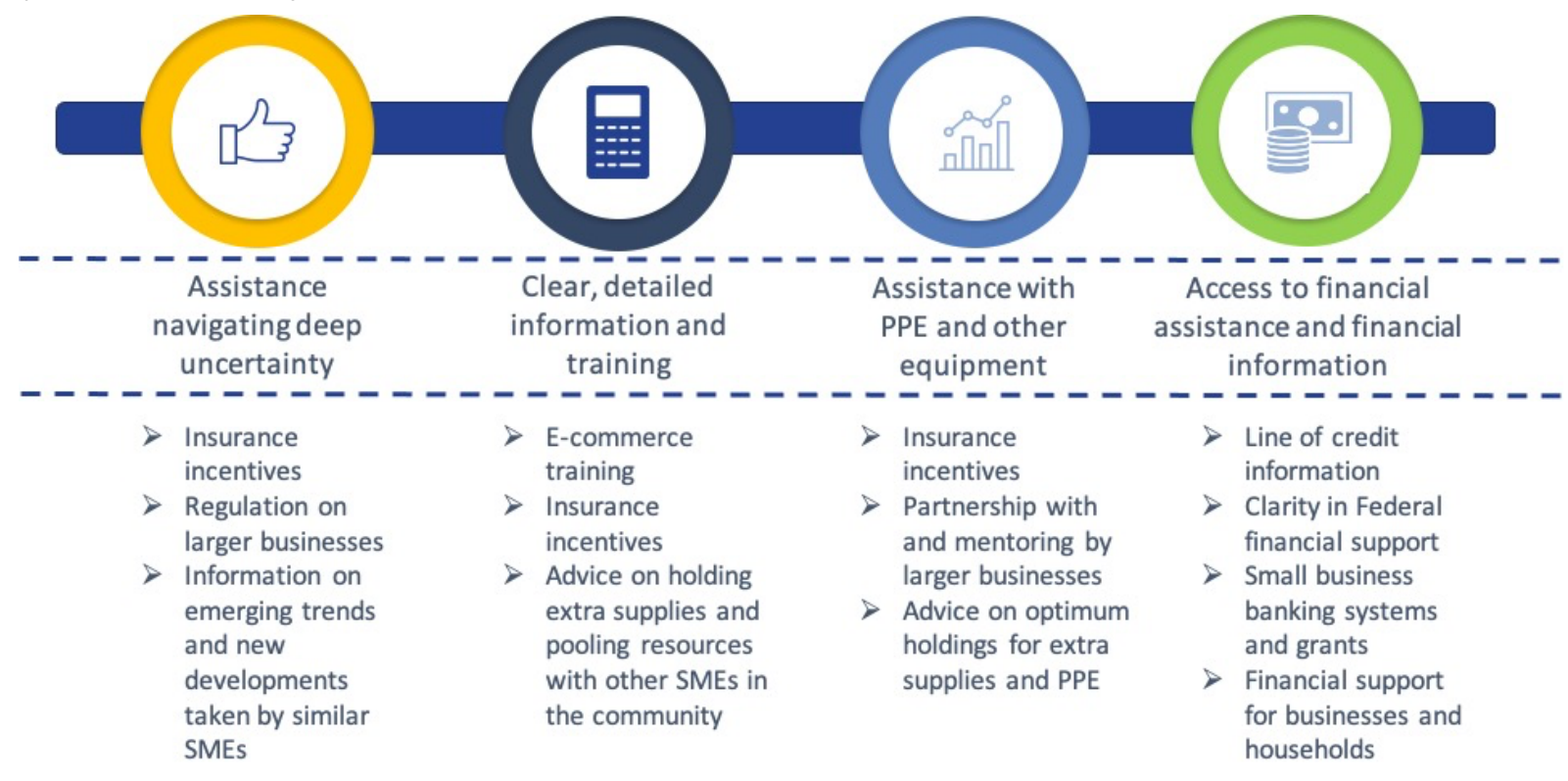

Figure 1 L. Iaentitiea categorıes от expressea neeas ana proposea solutions.

Finally, the survey asked respondents to indicate their level of agreement with the statements shown in Figure 13. Nearly $18 \%$ agreed with the statement about COVID-19 posing the greatest risk yet to their SME's survival. When asked to indicate agreement with the statement "impacts of COVID-19 will leave my business unable to cope with a natural disaster, should one occur in the next year," $27.1 \%$ strongly agreed, while $37 \%$ strongly disagreed. In addition, some $40 \%$ expressed some level of agreement with the statement "Stress in my home life from COVID-19 has created increased stress for my business."

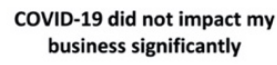$$
\text { (1) }
$$

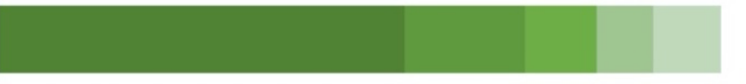

COVID-19 is/was the greatest risk to my SME's survival
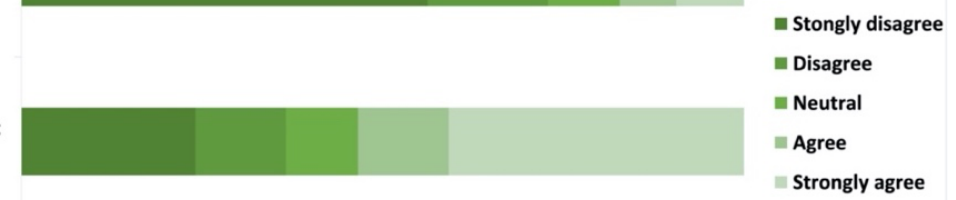

COVID-19 impacts leave my SME unable to cope with a natural disaster (in the next 12 months)

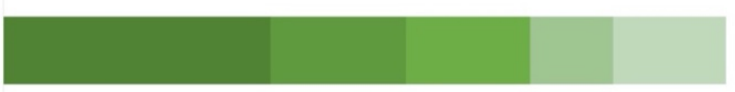

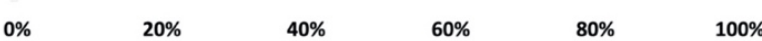

Figure 13. Agreement with key perception questions related to the impact of COVID-19. $n=192$ 


\section{Initial Findings: Summary}

Operating in a multi-risk environment with acute and chronic shocks and stressors requires that SMEs address potential complex events that arise from compound and/or cascading risks in the context of COVID-19. Being mindful of existing social vulnerabilities and their capacity to amplify the impacts of other events is critical. This section presents a summary of key initial findings.

- $31.6 \%$ of respondents experienced at least one natural disaster during this period.

- $68.8 \%$ of respondents implemented some adaptation/coping strategies for COVID-19, with $26 \%$ reporting the use of natural hazard mitigation strategies.

- $\quad 28.7 \%$ of respondents plan to adopt practices used during the COVID-19 pandemic in anticipation of future natural hazards.

- $\quad 75.3 \%$ of businesses are concerned about future complex events, of which $45.2 \%$ identify their concern as arising from natural disaster(s) and a subsequent wave of COVID-19 associated restrictions.

In agreement with Wave 1 findings, Wave 2 respondents indicated that four categories of resources and information would help them address and plan in the future for complex event risks. The specific potential solutions to meet these needs were highlighted by Wave 2 respondents are shown in Figure 12 .

\section{Future Research}

As of this writing, over a year has passed since the March 13, 2020 Federal Emergency Declaration concerning COVID-19. Many Americans report stress on their physical and mental health, with essential workers more than twice as likely to have received treatment from a mental health professional. ${ }^{12}$ The situation in the United States is improving. Between March 2020 and March 2021, Congress has approved several stimulus and relief packages, with businesses receiving over $\$ 700$ billion in forgivable loans. ${ }^{13}$ While the short-term benefits of stimulus payments to households and loans to small businesses are unclear, mitigating health concerns is key to economic recovery (Chetty et al. 2020). As of March 29, 2021, over 95 million people had received at least one vaccine dose, with over 52 million fully vaccinated. ${ }^{14}$

This survey constitutes the second data collection in a longitudinal effort to address SME Complex Event Resilience. This effort is aimed at providing relevant data to Federal partners and other entities in providing guidance to SMEs concerning: (1) mitigation planning for natural disasters during the pandemic and (2) disaster readiness strategies to cope with disruptions from the pandemic. Understanding the relative learning, agency, and flexibility of SME operators towards complex events remains relevant to processes, institutions, and policies (Helgeson et al., 2021b). ${ }^{15}$

Future data collected will focus on recovery trajectories and potential tradeoffs faced by SME operators as they plan for recovery and prepare for future complex event disruptions. As this longitudinal research effort continues, feedback and collaboration are welcome.

For more information, please contact: SMEResearch@nist.gov

\footnotetext{
${ }^{12}$ American Psychological Association (2021). Stress in America 2021: One year later, a new wave of pandemic health concerns. https://www.apa.org/news/press/releases/stress/2021/one-year-pandemic-stress. Accessed: 2021-03-30.

${ }_{13}$ U.S. Chamber of Commerce (2021). Everything You Need to Know About Coronavirus Federal Small Business Stimulus Aid Programs. https://uschamber.com/co/start/strategy/federal-small-business-stimulus-aid-program-guide. Accessed: 2021-03-30.

${ }_{14}$ CDC (2021). COVID-19 Vaccinations in the United States. https://covid.cdc.gov/covid-data-tracker/\#vaccinations. Accessed: 2021-03-30.

${ }^{15}$ Helgeson, J., J. Fung, and A. Roa-Henriquez. (2021b). "Rationally bounded in a storm of complex events: small businesses facing natural hazard resilience during a pandemic." Journal of Behavioral Economics for Policy. Vol. 4, COVID-19 Special Issue 3, 55-65.

https://sabeconomics.org/wordpress/wp-content/uploads/JBEP-4-S3-4.pdf
} 